\title{
Bidirectional tuning of microglia in the developing brain: From neurogenesis to neural circuit formation
}

Masaki Ueno ${ }^{\mathrm{a}, \mathrm{b} \dagger}$ and Toshihide Yamashita ${ }^{\mathrm{c}, \mathrm{d} \dagger}$

aDivision of Developmental Biology, Cincinnati Children's Hospital Medical Center, 3333 Burnet Avenue, Cincinnati, OH, 45229

bPrecursory Research for Embryonic Science and Technology (PRESTO), Japan

Science and Technology Agency (JST), 4-1-8 Honcho, Kawaguchi, Saitama, 332-

0012, Japan

${ }^{\mathrm{c}}$ Department of Molecular Neuroscience, Graduate School of Medicine, Osaka

University, 2-2 Yamadaoka, Suita-shi, Osaka 565-0871, Japan

${ }^{\mathrm{d}}$ Core Research for Evolutional Science and Technology (CREST), Japan Science and Technology Agency (JST), 5 Sanbancho, Chiyoda-ku, Tokyo, Japan

$\dagger$ Corresponding authors:

Toshihide Yamashita

E-mail: yamashita@molneu.med.osaka-u.ac.jp

Masaki Ueno

E-mail: ms-ueno@umin.ac.jp 


\begin{abstract}
The developing brain employs multi-step processes to construct neural circuitry. Recent studies have highlighted that microglia, traditionally known to be the resident immune cells in the brain, have essential roles in these processes, which range from neurogenesis to establishing synaptic connections. Microglia play bidirectional roles for maintaining proper circuitry: eliminating unnecessary cells, axons, and synapses, while supporting the neighboring ones. Although these processes are performed in different parts of the neuron, similar molecular mechanisms are possibly involved. This paper reviews recent progress on the knowledge of the roles of microglia in brain development, and further discusses the application of this knowledge in therapies for brain disorders and injuries.
\end{abstract}




\section{Introduction}

Microglia account for $3-12 \%$ of the cells in the central nervous system (CNS) and are commonly referred to as the brain's resident immune cells or tissue macrophages $[1,2]$. Microglia play diverse roles in pathological conditions, owing to their ability to dramatically change morphology and engage in inflammatory/repair responses $[3,4]$. Recent technological advances have revealed additional physiological functions of microglia in the healthy brain, particularly, during development where dramatic changes in neuronal circuitry occur daily. Here, we review the newly discovered role of microglia on brain development, and further discuss whether understanding these functions will aid in therapeutic treatment of diseases that target the CNS.

\section{Origin of microglia and their distribution}

Although the majority of cells in the brain are generated from neural progenitor cells, microglia are considered to be of monocyte/macrophage lineage because of their shared features. However, whether their origin is distinctly defined during development or recruited from monocytes in circulating blood has been a subject of debate $[5,6]$. This topic has recently been addressed by a study that used parabiosis to demonstrate that blood-derived monocytes are not provided to the adult brain and microglia can "self-renew" [7]. This implies that there is a specific, predetermined origin of microglia during development. Indeed, expression of microglial markers suggest that they are derived from the embryonic yolk sac [8], and more recent studies using genetic mice have shown direct evidence of this (Fig. 1a) [9-11]. In these studies, taking advantage of early markers such as RUNX1, a transcription factor, and CSF1R, a receptor for CSF1 (M-CSF), the lineage of microglia was traced using Runxl-CreER and Csflr-CreER;YFP reporter mice with tamoxifen injection to label 
yolk sac progenitor cells in a restricted developmental time window. It was demonstrated that microglia were derived from myeloid progenitors at around E7.5 and the cells were Myb-negative and PU.1-dependent Csf1-receptor-expressing progenitors, which are different from Myb-dependent hematopoietic stem cells that differentiate into other macrophages and monocytes $[9,10]$. The molecular profiles of these progenitors have been further identified [11].

In mice, cells migrate out of the yolk sac and infiltrate the brain at E9.5 (Fig. 1a and b) $[5,8-10,12,13]$. Although the route to infiltration is not yet completely understood, histological studies imply that these cells enter from the meninges, ventricles, and intracerebral blood vessels. Once in the brain, microglia gradually increase in number and colonize areas such as subcortical and cerebellar white matter in early postnatal days (Fig. 1d). These colonized regions are referred to as "fountains of microglia" in rodents [14-16] and humans [17,18] and are ultimately distributed to most brain regions presumably through migration and proliferation $[12,14,16]$.

The timely and locally invasive nature of microglia implicates that these cells play a critical role in brain development. In fact, recent reports have revealed that microglia are involved in the construction of neural circuits, which are assembled in multiple stages: neural progenitors proliferating around the ventricular zone (VZ), differentiated neurons migrating to specific areas, and neurons projecting axons to target areas and forming synapses (Fig.1).

\section{Bidirectional roles of microglia in neurogenesis}

During the course of brain development, significant numbers of neurons and glial cells (astrocytes and oligodendrocytes) must be precisely generated from neural progenitor cells. Interestingly, microglia have been observed in the neuroepithelium 
where progenitor cells proliferate $[5,6,8,9,19]$, implying a role for microglia in neurogenesis (Fig. 1b and c). Microglia have been shown to exert trophic effects in vitro. For example, cultured embryonic cortical cells of PU.1 $1^{-/}$mice lacking microglia demonstrate no change in cell survival and neurogenesis of progenitor cells, but exhibit reduced proliferation and astrogenesis [19]. A number of factors known to be expressed in microglia, such as BDNF, FGF2, and IGF1 $[14,20]$, may be involved in this process. In vivo, a decline in microglia is correlated with reduced histogenesis of the brain in $\mathrm{Csflr}^{-/}$mice [21]. This study implies a role for microglia in neurogenesis; however, whether this is a direct effect of microglia remains unclear.

Conversely, it was found an opposite role of microglia in that they can restrict the number of neural progenitor cells in vivo [22]. This group observed prominent numbers of microglia in the VZ and subventricular zone (SVZ). In these zones, nondying progenitors phagocytosed by microglia gradually increased, and this was correlated with reduction in the number of progenitor cells. Deactivating or eliminating microglia by tetracycline or liposomal clodronate, respectively, significantly increased the number of progenitor cells, whereas activating microglia by lipopolysaccharide (LPS) decreased the number of these cells. Therefore, the authors concluded that microglia regulate the number of progenitor cells mainly through phagocytosis. This can be related to the phagocytosis of new born cells in the hippocampal proliferative zone [23].

From these results, it has been postulated that microglia play bidirectional roles in neurogenesis by supporting cell generation and removing unnecessary cells.

\section{Microglia control the survival and death of developing neurons}

The regulation of neuronal number is essential to the construction of neural circuitry. 
In the developing CNS, cell death occurs extensively to eliminate unhealthy and misconnected neurons [24]. Survival of neurons is presumably maintained by factors derived from target or connected neurons and glial cells. While cell death was thought to be determined autonomously within the neuronal circuit, recent findings indicate that microglia might also be involved (Fig. 1d). In the developing retina, proNGF secreted from microglia have been shown to induce cell death via P75 neurotrophin receptor [25]. In another case, TNF $\alpha$ derived from macrophages adjacent to the spinal cord was shown to commit motor neurons to die [26]. These studies suggest that microglia promote cell death through secreted molecules. One other mechanism that induces cell death is phagocytosis [29,30], which is classically considered to be just the process of eliminating dying cells in both the normal and damaged developing brain [31]. In the postnatal cerebellum, many dying Purkinje cells are phagocytosed by microglia. Eliminating microglia by liposomal clodronate or inhibition of NADPH oxidase can significantly reduce Purkinje cell death [32]. This indicates that microglia aid the process of cell death by engulfment and release of superoxide ions. Although the studies above should be carefully interpreted since they were all conducted in vitro in which microglia may easily change their activity (to toxic, in this case) $[27,28]$, the following in vivo study demonstrated that DAP12-CD11b-ROS signaling contributed to developmental death of postnatal hippocampal neurons [33], as similarly observed in progenitor cells [22]. Future work is required in order to elucidate whether microglia facilitate cell death, just engulf dying cells, or do both.

While the mechanism by which microglia recognize target cells ("find me" signal), engulf them ("eat me" signal), and induce cell death still remains unclear, some clues have surfaced. Microglia phagocytize healthy neurons in inflammatory conditions and use a similar mechanism to engulf apoptotic cells [30,34,35]. Phosphatidylserine (PS) 
exposed on neurons is bound by MFG-E8, which are then phagocytized via the vitronectin receptor on microglia. In this context, phagocytizing glial cells (not categorized as microglia/macrophages) likely use Jedi-1 and MEGF10, previously reported as CED-1 in C. elegans [36] and Draper in Drosophila [37] respectively, for phagocytosis in developing dorsal root ganglia [38,39]. Again, most of these experiments were conducted in vitro and not tested in microglia in the developing brain.

We recently found an opposite function in which microglia can promote the survival of specific neurons in the postnatal cortex (Fig. 1d) [14]. The first aim was to determine the role of postnatal amoeboid microglia which typically accumulated in the white matter $[15,16,40,41]$. To address this, we inactivated or eliminated microglia by using minocycline treatment, CD11b-DTR transgenic mice, and $\mathrm{Cx}_{3} \mathrm{Cr}^{-}$ ${ }^{-}$mice. Interestingly, all of these modifications led to increased apoptosis in layer $\mathrm{V}$ neurons at P3-5. This time window correlated with the period when microglia accumulate near the axons of layer $\mathrm{V}$ neurons. We also identified microglia-derived IGF1 as a survival factor. Additional factors might be involved in the effects of microglia since IGF1 only partially supported neuronal survival.

Collectively, microglia locally patrolling eliminate neurons showing abnormal signs ("eat me"/“kill me" signals), and support the survival of surrounding healthy neurons not expressing abnormal signs (or expressing “don't eat me” signals). These processes would result in preventing secondary necrosis and inflammation, and help maintain homeostasis.

Microglia engage in maintenance of axons and synapses during neural circuit formation 
Microglia likely perform similar functions with respect to axons and synapses during neuronal wiring. In the initial phase, axons robustly project to a target area and improper connections are pruned in a degenerative or retractive manner [42]. In Drosophila, glial cells surround axons from mushroom body, engulfing degenerating ones [43]. Some neuronal signal is responsible for attracting glial cells to these dying axons and glial cells seem to facilitate the degenerative process [43]. The engulfment occurs through Draper and Ced-6, which are also known to be involved in enveloping apoptotic cells [44]. This implicates that apoptotic cells and degenerating axons are removed by a similar molecular mechanism. In the mammalian system, microglia has a capacity for engulfing axonal debris [45] and microglia-engulfing axonal materials are observed where pruning occurs $[14,16,42]$. Despite their close correlation, the role in axon pruning is still unknown; microglia presumptively engulfs axonal material, but it is not clear whether they facilitate pruning (Fig. 1d).

Along with elimination, microglia may have a supportive effect on axon growth and guidance $[41,46]$. During the postnatal period, amoeboid-shaped microglia in the white matter where axons are growing express axonal growth factors, such as IGF1 [14], thrombospondin [47,48], and NT3 [49]. They also express guidance molecules, such as Slit, Netrin [50], Sema7a [51], and RGMa [52] in the injured CNS. However, there is no direct evidence in vivo, that microglia support neighboring axons during axonal pruning (Fig. 1d).

The role of microglia on synapse formation is a topic of much interest (Fig. 1e) [53]. This comes with the finding that microglia are highly active with motile processes, which was discovered by using the combination of microglia-specific EGFP-expressing mice $\left(C x 3 c r 1^{G F P /+}\right)$ and two-photon microscopy [54,55]. Subsequently, in vivo imaging and electron microscopic analyses revealed that 
microglia frequently contact synapses in a neuronal activity-dependent manner, suggesting a role for microglia in synaptic remodeling [56]. During development, synapses are initially generated superabundantly and then pruned to refine a circuit. In the juvenile visual cortex, dendritic spines are contacted by microglia, and a quarter of them disappear after the contact [57]. Moreover, deprivation of visual input results in increased contact and synapse-like inclusions in microglia. This suggests that microglia eliminate excess synapses to remodel connections. Consistent with this, pre- and postsynaptic molecules have been detected in microglia in the postnatal hippocampus, and decreases in microglial number in $C x 3 \mathrm{crl}$-knockout mice increase the number of dendritic spines [58]. Similar correlations have been observed in the postnatal somatosensory cortex [59]. While the molecular mechanism still remains unclear, CX3CL1 (fractalkine)-CX3CR1 signaling may be required for migration into the microcircuit, or for locally targeting the synapses.

Components of the classical complement cascade, C3 abundant in synapses, and its receptor CR3 expressed in microglia, have been shown to be involved in the phagocytic process of synapses in the postnatal retinogeniculate circuit [60]. Microglia engulf presynaptic materials of weak input side when binocular input to the dorsal lateral geniculate nucleus (dLGN) is being remodeled. Engulfed synapses are decreased and refinement of binocular input to the dLGN is impaired in C3- and CR3knockout mice $[60,61]$. It remains unknown whether microglia selectively detect weak synapses via the C3/CR3 system, and this system is generally used in other brain regions.

While the studies above suggest that microglia play an essential role in engulfing unnecessary synapses, these cells may also maintain required synapses given their bidirectional role in other developmental processes. Indeed, contacted pre- and post- 
synaptic sites alter their size by both growing and shrinking, and not all the spines are eliminated [57]. Other properties of microglia have been uncovered in the optic tectum of larval zebrafish. Microglial processes were directed to contact active neurons, requiring membrane depolarization-activated pannexin-1 hemichannels in neurons that can release ATP etc., and the contact downregulated neuronal activity [62]. If this is the case in all neurons, microglia would use molecular mechanisms to select specific synapses to contact, eliminate, or preserve (Fig. 1e).

\section{How do microglia migrate into and within the brain: unresolved questions}

The mechanism underlying microglial migration is an unresolved question. Although microglia distribute ubiquitously in the adult brain, a variety of developmental tasks are often specialized in each brain region. Therefore, oriented migration and recruitment in a timely and local manner are important processes. Nevertheless, it remains unknown how microglia recognize the brain and their final location (Fig. 1). Although the blood-brain barrier (BBB) is immature enough for cells to enter during development, it is clear that microglia do not just randomly enter the brain from the vasculature. For example, amoeboid microglia are precisely controlled to accumulate in the postnatal white matter $[15,16,40]$. We have observed that microglia likely invade the postnatal forebrain from the midline, ventricle, or choroid plexus, but it is unclear how these cells are recruited in such a precise time window and location. Interestingly, microglia exogenously introduced into the blood stream specifically target to the brain [63]. This implies that some specific cues are involved in the relocation process. In the experimental autoimmune encephalomyelitis (EAE) model, autoreactive Th17 cells enter the CNS from a specific lumbar region through CCL20-CCR6 signaling, suggesting that some gateways are located in the 
CNS that render the invasion of immune cells [64]. Interestingly, CCL20 expression is higher in this region even in the healthy CNS. From this view, it will be possible to imagine that microglia might also use a similar gating system.

The candidates involved in microglia migration could include chemokines, ATPP2X4/P2Y12 signaling, and glutamate [65-67]. For example, the panther mutant in zebrafish, which lacks the M-CSF receptor gene, shows delayed invasion of microglia into the brain [13]. Likewise, microglia are reduced in Csf1 $r^{--}$mice [9,21]; however, it is still unresolved whether this signal is required to migrate out from the yolk sac, to invade the brain, or for just microglia production in the yolk sac. Some other chemokines can be excluded as the candidates. CCR2 has been shown to be expressed in monocytes but not in microglia and $C \mathrm{cr} 2^{-/-}$mutants do not show reduced microglia numbers $[11,68,69] . C x 3 c r 1^{G F P / G F P}, C \mathrm{Crl}^{-/}$, and $\mathrm{Cxcr}^{-/}$do not alter entry of microglia into the brain $[11,14]$. Similarly, $P 2 y 12^{-/}$mice do not exhibit a reduction of microglia in the adult brain [66], although it have not yet been thoroughly examined in development. One recent study found that VEGFR1-NOX2 signaling is required, in part, for microglial migration from the ventricle to the SVZ [65], but the entire mechanism still remains unknown.

One other possible mechanism is that microglia are attracted by degenerating axons or cells; however, the "find me" molecule has not yet been tested in this context. Importantly, these attracting molecules may also be involved in local migration and motility that would be essential for the other tasks in targeting specific neurons and synapses. Thus, it will be intriguing to investigate the mechanism underlying directional migration. Lastly, if we know the mechanism underlying migration, we may be able to control the number of infiltration microglia/macrophages in CNS diseases. 
How are the variable functions of microglia regulated? - application to CNS disease

One key advantage to understanding the variable roles of microglia would be to clarify the multiple functional states and heterogenous populations of microglia, that apparently contribute to normal brain development and function. For example, only $40 \%$ of microglia are derived from Hoxb8-expressing hematopoietic cells, the mutation of which leads to abnormal grooming behavior [12]. However, it is not clear how these heterogeneous populations and functions emerge. Microglia have been traditionally classified as a "resting" state, which have a ramified morphology (or recently "surveying" microglia [70]), and an "activated" state, showing profound changes in shape and gene expression, particularly in pathological conditions. Even if we use this simple classification, it cannot explain the diverse set of functions. For example, "activated" microglia have bidirectional functions, exerting both toxic and trophic effects onto neurons [14,32]. Other classifications, M1 (classic) and M2 (alternative) states, which applied from macrophage literature [71], is not easily applied to the healthy brain and its development. Indeed, microglia in the P3 mouse brain highly express both M1 (iNOS and TNF $\alpha$ ) and M2 genes (Arginase-1), compared to adult microglia [28]. Even in diseased conditions, microglia behave differently from macrophage/monocytes [72,73]. Tissue macrophages, including microglia, are considered to be M2 but this rigid view may lead to misunderstandings about heterogeneous functions of microglia.

Therefore, it should be clear that the biggest problem posed in this field is the difficulty in dissecting out the multiple faces of microglia: what activation and deactivation are, in which state phagocytosis, toxic, and trophic functions occur, as 
well as how these states are regulated. These multiple factors hinder our understanding of the real roles of microglia. It would be important to understand the mechanism that transits the properties of microglia (i.e., resting (surveying) vs. activated and M1 vs. M2). However, because this simple classification cannot demonstrate whole properties of microglia, we also need to explore the function of each gene expressed in specific microglial populations. This may lead to a more direct understanding of the variable functions.

The tools used to specifically modify microglia in vivo are critical factors in advancing in this field. Some drugs frequently used, such as minocycline and LPS, are not specific to microglia. Some genetic and chemical approaches to ablate microglia (liposome clodronate, CD11b HSVTK mice, and CD11b-DTR mice [27]) will ablate heterogeneous microglia, thereby eliminating each individual function. Thus, tools to selectively delete specific genes such as Cx3crl-cre, Cx3crl-creER

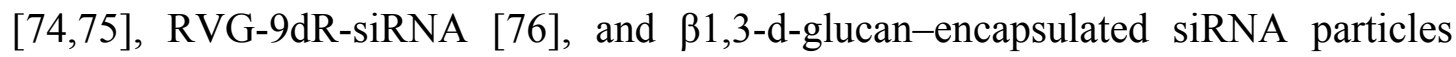
[77], will be more promising approaches. It will be intriguing if these specific deletions can regulate each microglial function such as phagocytosis, or states of activation. These technologies will pave a new way to expand our knowledge of microglia.

As reviewed here, microglia have diverse roles even in the normal brain and its development. This suggests that disruption of microglia function can lead to CNS disorders. For example, obsessive grooming behavior, a model of trichotillomania, can be observed following Hoxb8 deletion in microglia [12]. Mutation of the Sod1 gene in microglia promotes late progression of diseases related to amyotrophic lateral sclerosis (ALS) [78]. Phagocytic deficiency in $\mathrm{Mecp}^{-/-}$microglia attributes to the pathology in a mouse model of Rett syndrome, an X-linked autism spectrum disorder, 
suggesting that clearance of debris is essential for normal brain function [79]. Many reports have further demonstrated that microglia engage in tissue defense and repair by their diverse functions such as phagocytosis, cell death induction, neuroprotection, axonal modulation, and synapse monitoring. Thus, understanding the molecular mechanisms of each function in brain development will help lead to the establishment of a therapeutic method to regulate microglial functions and attenuate symptoms of CNS diseases.

\section{Acknowledgements}

The works in Yamashita lab are supported by a grant for Core Research for Evolutional Science and Technology (CREST) from the Japan Science and Technology Agency (JST). M.U. was supported by a Grant-in-Aid for Young Scientists (B) and Postdoctoral Fellowships for Research Abroad from the Japan Society for the Promotion of Science (JSPS), and Kanae Foundation for the Promotion of Medical Science, and is supported by Precursory Research for Embryonic Science and Technology (PRESTO) from JST. We apologize that many important publications have not been cited due to space limitations. 
References

1. Lawson LJ, Perry VH, Dri P, Gordon S: Heterogeneity in the distribution and morphology of microglia in the normal adult mouse brain. Neuroscience 1990, 39:151-170.

2. Pelvig DP, Pakkenberg H, Stark AK, Pakkenberg B: Neocortical glial cell numbers in human brains. Neurobiol Aging 2008, 29:1754-1762.

3. Kettenmann H, Hanisch UK, Noda M, Verkhratsky A: Physiology of microglia. Physiol Rev 2011, 91:461-553.

4. Ransohoff RM, Perry VH: Microglial physiology: unique stimuli, specialized responses. Annu Rev Immunol 2009, 27:119-145.

5. Chan WY, Kohsaka S, Rezaie P: The origin and cell lineage of microglia: new concepts. Brain Res Rev 2007, 53:344-354.

6. Kaur C, Hao AJ, Wu CH, Ling EA: Origin of microglia. Microsc Res Tech 2001, 54:2-9.

7. Ajami B, Bennett JL, Krieger C, Tetzlaff W, Rossi FM: Local self-renewal can sustain CNS microglia maintenance and function throughout adult life. Nat Neurosci 2007, 10:1538-1543.

8. Alliot F, Godin I, Pessac B: Microglia derive from progenitors, originating from the yolk sac, and which proliferate in the brain. Brain Res Dev Brain Res 1999, 117:145-152.

9. Ginhoux F, Greter M, Leboeuf M, Nandi S, See P, Gokhan S, Mehler MF, Conway SJ, Ng LG, Stanley ER, et al.: Fate mapping analysis reveals that adult microglia derive from primitive macrophages. Science 2010, 330:841845.

10. Schulz C, Gomez Perdiguero E, Chorro L, Szabo-Rogers H, Cagnard N, Kierdorf K, Prinz M, Wu B, Jacobsen SE, Pollard JW, et al.: A lineage of myeloid cells independent of Myb and hematopoietic stem cells. Science 2012, 336:86-90.

11. Kierdorf K, Erny D, Goldmann T, Sander V, Schulz C, Perdiguero EG, Wieghofer $\mathrm{P}$, Heinrich A, Riemke $\mathrm{P}$, Hölscher C, et al.: Microglia emerge from erythromyeloid precursors via Pu.1- and Irf8-dependent pathways. Nat Neurosci 2013, 16:273-280.

12. Chen SK, Tvrdik P, Peden E, Cho S, Wu S, Spangrude G, Capecchi MR: Hematopoietic origin of pathological grooming in Hoxb8 mutant mice. Cell 2010, 141:775-785.

13. Herbomel P, Thisse B, Thisse C: Zebrafish early macrophages colonize cephalic mesenchyme and developing brain, retina, and epidermis through a M-CSF receptor-dependent invasive process. Dev Biol 2001, 238:274-288.

14. Ueno M, Fujita Y, Tanaka T, Nakamura Y, Kikuta J, Ishii M, Yamashita T: Layer $\mathrm{V}$ cortical neurons require microglial support for survival during postnatal development. Nat Neurosci 2013, 16:543-551.

15. Milligan CE, Cunningham TJ, Levitt P: Differential immunochemical markers reveal the normal distribution of brain macrophages and microglia in the developing rat brain. J Comp Neurol 1991, 314:125135.

16. Hristova M, Cuthill D, Zbarsky V, Acosta-Saltos A, Wallace A, Blight K, Buckley $\mathrm{SM}$, Peebles $\mathrm{D}$, Heuer $\mathrm{H}$, Waddington $\mathrm{SN}$, et al.: Activation and 
deactivation of periventricular white matter phagocytes during postnatal mouse development. Glia 2010, 58:11-28.

17. Monier A, Evrard P, Gressens P, Verney C: Distribution and differentiation of microglia in the human encephalon during the first two trimesters of gestation. J Comp Neurol 2006, 499:565-582.

18. Verney C, Monier A, Fallet-Bianco C, Gressens P: Early microglial colonization of the human forebrain and possible involvement in periventricular white-matter injury of preterm infants. J Anat 2010, 217:436-448.

19. Antony JM, Paquin A, Nutt SL, Kaplan DR, Miller FD: Endogenous microglia regulate development of embryonic cortical precursor cells. $J$ Neurosci Res 2011, 89:286-298.

20. Nakajima K, Kohsaka S: Microglia: neuroprotective and neurotrophic cells in the central nervous system. Curr Drug Targets Cardiovasc Haematol Disord 2004, 4:65-84.

21. Erblich B, Zhu L, Etgen AM, Dobrenis K, Pollard JW: Absence of colony stimulation factor-1 receptor results in loss of microglia, disrupted brain development and olfactory deficits. PLoS One 2011, 6:e26317.

22. Cunningham CL, Martínez-Cerdeño V, Noctor SC: Microglia regulate the number of neural precursor cells in the developing cerebral cortex. $J$ Neurosci 2013, 33:4216-4233.

23. Sierra A, Encinas JM, Deudero JJ, Chancey JH, Enikolopov G, OverstreetWadiche LS, Tsirka SE, Maletic-Savatic M: Microglia shape adult hippocampal neurogenesis through apoptosis-coupled phagocytosis. Cell Stem Cell 2010, 7:483-495.

24. Oppenheim RW, Johnson JE: Programmed cell death and neurotrophic factors. Edited by Squire LR, Bloom FE, McConnell SK, Roberts JL, Spitzer NC, Zigmond MJ: Academic press; 2003.

25. Frade JM, Barde YA: Microglia-derived nerve growth factor causes cell death in the developing retina. Neuron 1998, 20:35-41.

26. Sedel F, Béchade C, Vyas S, Triller A: Macrophage-derived tumor necrosis factor alpha, an early developmental signal for motoneuron death. $J$ Neurosci 2004, 24:2236-2246.

27. Hellwig $S$, Heinrich $A$, Biber $K$ : The brain's best friend: microglial neurotoxicity revisited. Front Cell Neurosci 2013, 7:71.

28. Crain JM, Nikodemova M, Watters JJ: Microglia express distinct M1 and M2 phenotypic markers in the postnatal and adult central nervous system in male and female mice. J Neurosci Res 2013, 91:1143-1151.

29. Mallat M, Marín-Teva JL, Chéret C: Phagocytosis in the developing CNS: more than clearing the corpses. Curr Opin Neurobiol 2005, 15:101-107.

30. Brown GC, Neher JJ: Eaten alive! Cell death by primary phagocytosis: 'phagoptosis'. Trends Biochem Sci 2012, 37:325-332.

31. Ueno M, Katayama K, Yamauchi H, Yasoshima A, Nakayama H, Doi K: Repair process of fetal brain after 5-azacytidine-induced damage. Eur $\mathrm{J}$ Neurosci 2006, 24:2758-2768.

32. Marín-Teva JL, Dusart I, Colin C, Gervais A, van Rooijen N, Mallat M: Microglia promote the death of developing Purkinje cells. Neuron 2004, 41:535-547. 
33. Wakselman S, Béchade C, Roumier A, Bernard D, Triller A, Bessis A: Developmental neuronal death in hippocampus requires the microglial CD11b integrin and DAP12 immunoreceptor. J Neurosci 2008, 28:8138-8143.

34. Fricker M, Neher JJ, Zhao JW, Théry C, Tolkovsky AM, Brown GC: MFG-E8 mediates primary phagocytosis of viable neurons during neuroinflammation. J Neurosci 2012, 32:2657-2666.

35. Neher JJ, Neniskyte U, Zhao JW, Bal-Price A, Tolkovsky AM, Brown GC: Inhibition of microglial phagocytosis is sufficient to prevent inflammatory neuronal death. J Immunol 2011, 186:4973-4983.

36. Reddien PW, Horvitz HR: The engulfment process of programmed cell death in caenorhabditis elegans. Annu Rev Cell Dev Biol 2004, 20:193221.

37. Freeman MR, Delrow J, Kim J, Johnson E, Doe CQ: Unwrapping glial biology: Gcm target genes regulating glial development, diversification, and function. Neuron 2003, 38:567-580.

38. Wu HH, Bellmunt E, Scheib JL, Venegas V, Burkert C, Reichardt LF, Zhou Z, Fariñas I, Carter BD: Glial precursors clear sensory neuron corpses during development via Jedi-1, an engulfment receptor. Nat Neurosci 2009, 12:1534-1541.

39. Scheib JL, Sullivan CS, Carter BD: Jedi-1 and MEGF10 signal engulfment of apoptotic neurons through the tyrosine kinase Syk. J Neurosci 2012, 32:13022-13031.

40. Ling EA, Ng YK, Wu CH, Kaur C: Microglia: its development and role as a neuropathology sensor. Prog Brain Res 2001, 132:61-79.

41. Streit WJ: Microglia and macrophages in the developing CNS. Neurotoxicology 2001, 22:619-624.

42. Luo L, O'Leary DD: Axon retraction and degeneration in development and disease. Annu Rev Neurosci 2005, 28:127-156.

43. Awasaki T, Ito K: Engulfing action of glial cells is required for programmed axon pruning during Drosophila metamorphosis. Curr Biol 2004, 14:668-677.

44. Awasaki T, Tatsumi R, Takahashi K, Arai K, Nakanishi Y, Ueda R, Ito K: Essential role of the apoptotic cell engulfment genes draper and ced6 in programmed axon pruning during Drosophila metamorphosis. Neuron 2006, 50:855-867.

45. Tanaka T, Ueno M, Yamashita T: Engulfment of axon debris by microglia requires p38 MAPK activity. J Biol Chem 2009, 284:21626-21636.

46. Kaur C, Dheen ST, Ling EA: From blood to brain: amoeboid microglial cell, a nascent macrophage and its functions in developing brain. Acta Pharmacol Sin 2007, 28:1087-1096.

47. Chamak B, Morandi V, Mallat M: Brain macrophages stimulate neurite growth and regeneration by secreting thrombospondin. J Neurosci Res 1994, 38:221-233.

48. Chamak B, Dobbertin A, Mallat M: Immunohistochemical detection of thrombospondin in microglia in the developing rat brain. Neuroscience 1995, 69:177-187. 
49. Elkabes S, DiCicco-Bloom EM, Black IB: Brain microglia/macrophages express neurotrophins that selectively regulate microglial proliferation and function. J Neurosci 1996, 16:2508-2521.

50. Wehrle R, Camand E, Chedotal A, Sotelo C, Dusart I: Expression of netrin-1, slit-1 and slit-3 but not of slit-2 after cerebellar and spinal cord lesions. Eur J Neurosci 2005, 22:2134-2144.

51. Pasterkamp RJ, Peschon JJ, Spriggs MK, Kolodkin AL: Semaphorin 7A promotes axon outgrowth through integrins and MAPKs. Nature 2003, 424:398-405.

52. Kitayama M, Ueno M, Itakura T, Yamashita T: Activated microglia inhibit axonal growth through RGMa. PLoS One 2011, 6:e25234.

53. Tremblay M, Stevens B, Sierra A, Wake H, Bessis A, Nimmerjahn A: The role of microglia in the healthy brain. J Neurosci 2011, 31:16064-16069.

54. Nimmerjahn A, Kirchhoff F, Helmchen F: Resting microglial cells are highly dynamic surveillants of brain parenchyma in vivo. Science 2005, 308:1314-1318.

55. Davalos D, Grutzendler J, Yang G, Kim JV, Zuo Y, Jung S, Littman DR, Dustin $\mathrm{ML}$, Gan WB: ATP mediates rapid microglial response to local brain injury in vivo. Nat Neurosci 2005, 8:752-758.

56. Wake H, Moorhouse AJ, Jinno S, Kohsaka S, Nabekura J: Resting microglia directly monitor the functional state of synapses in vivo and determine the fate of ischemic terminals. J Neurosci 2009, 29:39743980.

57. Tremblay M, Lowery RL, Majewska AK: Microglial interactions with synapses are modulated by visual experience. PLoS Biol 2010, 8:e1000527.

58. Paolicelli RC, Bolasco G, Pagani F, Maggi L, Scianni M, Panzanelli P, Giustetto M, Ferreira TA, Guiducci E, Dumas L, et al.: Synaptic pruning by microglia is necessary for normal brain development. Science 2011, 333:1456-1458.

59. Hoshiko M, Arnoux I, Avignone E, Yamamoto N, Audinat E: Deficiency of the microglial receptor CX3CR1 impairs postnatal functional development of thalamocortical synapses in the barrel cortex. $J$ Neurosci 2012, 32:15106-15111.

60. Schafer DP, Lehrman EK, Kautzman AG, Koyama R, Mardinly AR, Yamasaki R, Ransohoff RM, Greenberg ME, Barres BA, Stevens B: Microglia sculpt postnatal neural circuits in an activity and complement-dependent manner. Neuron 2012, 74:691-705.

61. Stevens B, Allen NJ, Vazquez LE, Howell GR, Christopherson KS, Nouri N, Micheva KD, Mehalow AK, Huberman AD, Stafford B, et al.: The classical complement cascade mediates CNS synapse elimination. Cell 2007, 131:1164-1178.

62. Li Y, Du XF, Liu CS, Wen ZL, Du JL: Reciprocal regulation between resting microglial dynamics and neuronal activity in vivo. Dev Cell 2012, 23:1189-1202.

63. Sawada M, Imai F, Suzuki H, Hayakawa M, Kanno T, Nagatsu T: Brainspecific gene expression by immortalized microglial cell-mediated gene transfer in the mammalian brain. FEBS Lett 1998, 433:37-40. 
64. Arima Y, Harada M, Kamimura D, Park JH, Kawano F, Yull FE, Kawamoto T, Iwakura Y, Betz UA, Márquez G, et al.: Regional neural activation defines a gateway for autoreactive $T$ cells to cross the blood-brain barrier. Cell 2012, 148:447-457.

65. Lelli A, Gervais A, Colin C, Chéret C, Ruiz de Almodovar C, Carmeliet P, Krause KH, Boillée $S$, Mallat $M$ : The NADPH oxidase Nox2 regulates VEGFR1/CSF-1R-mediated microglial chemotaxis and promotes early postnatal infiltration of phagocytes in the subventricular zone of the mouse cerebral cortex. Glia 2013, 61:1542-1555.

66. Haynes SE, Hollopeter G, Yang G, Kurpius D, Dailey ME, Gan WB, Julius D: The P2Y12 receptor regulates microglial activation by extracellular nucleotides. Nat Neurosci 2006, 9:1512-1519.

67. Liu GJ, Nagarajah R, Banati RB, Bennett MR: Glutamate induces directed chemotaxis of microglia. Eur J Neurosci 2009, 29:1108-1118.

68. Mizutani M, Pino PA, Saederup N, Charo IF, Ransohoff RM, Cardona AE: The fractalkine receptor but not CCR2 is present on microglia from embryonic development throughout adulthood. J Immunol 2012, 188:29-36.

69. Mildner A, Schmidt H, Nitsche $M$, Merkler D, Hanisch UK, Mack M, Heikenwalder M, Brück W, Priller J, Prinz M: Microglia in the adult brain arise from Ly-6ChiCCR2+ monocytes only under defined host conditions. Nat Neurosci 2007, 10:1544-1553.

70. Hanisch UK, Kettenmann $\mathrm{H}$ : Microglia: active sensor and versatile effector cells in the normal and pathologic brain. Nat Neurosci 2007, 10:13871394.

71. Sica A, Mantovani A: Macrophage plasticity and polarization: in vivo veritas. J Clin Invest 2012, 122:787-795.

72. Ajami B, Bennett JL, Krieger C, McNagny KM, Rossi FM: Infiltrating monocytes trigger EAE progression, but do not contribute to the resident microglia pool. Nat Neurosci 2011, 14:1142-1149.

73. Shechter R, London A, Varol C, Raposo C, Cusimano M, Yovel G, Rolls A, Mack $M$, Pluchino $S$, Martino $G$, et al.: Infiltrating blood-derived macrophages are vital cells playing an anti-inflammatory role in recovery from spinal cord injury in mice. PLoS Med 2009, 6:e1000113.

74. Wolf Y, Yona S, Kim KW, Jung S: Microglia, seen from the CX3CR1 angle. Front Cell Neurosci 2013, 7:26.

75. Goldmann T, Wieghofer P, Müller PF, Wolf Y, Varol D, Yona S, Brendecke SM, Kierdorf K, Staszewski O, Datta M, et al.: A new type of microglia gene targeting shows TAK1 to be pivotal in CNS autoimmune inflammation. Nat Neurosci 2013.

76. Kim SS, Ye C, Kumar P, Chiu I, Subramanya S, Wu H, Shankar P, Manjunath N: Targeted delivery of siRNA to macrophages for anti-inflammatory treatment. Mol Ther 2010, 18:993-1001.

77. Aouadi M, Tesz GJ, Nicoloro SM, Wang M, Chouinard M, Soto E, Ostroff GR, Czech MP: Orally delivered siRNA targeting macrophage Map4k4 suppresses systemic inflammation. Nature 2009, 458:1180-1184.

78. Boillée S, Yamanaka K, Lobsiger CS, Copeland NG, Jenkins NA, Kassiotis G, Kollias G, Cleveland DW: Onset and progression in inherited ALS 
determined by motor neurons and microglia. Science 2006, 312:13891392.

79. Derecki NC, Cronk JC, Lu Z, Xu E, Abbott SB, Guyenet PG, Kipnis J: Wild-type microglia arrest pathology in a mouse model of Rett syndrome. Nature 2012, 484:105-109.

\section{Annotation}

$10^{*}$ This study demonstrates that some of tissue macrophages including microglia are derived from specific lineage in yolk sac; Myb-independent, PU.1-dependent, and Csfl-receptor-expressing lineage, which can be distinguished with Myb-dependent hematopoietic stem cell-derived macrophages/monocytes. This concludes that microglia has a specific origin different from definitive hematopoietic stem cells.

$11^{*}$ Following the study in [10], the authors found several differentiation steps of microglia in the yolk sac and specifically c-kit-positive cells as a precursor. They further demonstrated that Irf8 and MMPs are required for microglial development and expansion in the brain.

14** Using different methods to modulate microglial function in vivo, the authors demostrate that postnatal microglia have a role to maintain the survival of specific neurons. This is the first report showing a trophic effect of microglia on neurons during brain development.

$22 *$ This study show that microglia engulf neural precursor cells and may regulate the proper size of precursor pool during development. This suggests that microglia do not just clean cellular debris up, but also actively control the number of cells via phagocytosis.

$60 * *$ This study demonstrate that microglia actively engage in synapse elimination during neural circuit formation, and reveal that CR3/C3 signaling is involved in this process. This study is extended from [61], and is the first report showing the key molecules involved in synaptic engulfment by microglia.

$62 * *$ This study reveals that neuronal activity steers patrolling microglial processes to contact neurons using pannexin-1 hemichannels, and eventually the contact reduces neuronal activity. This highlights a novel physiological role of microglia to regulate homeostasis of neural circuit.

64** This study demonstrate that, in an EAE model, autoreactive immune cells infiltrate the CNS from a specific site through upregulation of chemokine CCL20 induced by sypathetic nerve activation. This proposes a system that creates an entry site of immune-related cells in the brain.

65* The authors show that NOX2 and VEGF signaling is required for infiltration of postnatal microglia around the ventricle. This study proposes a novel field to investigate the mechanism of microglial migration and infiltration in a developing brain. 
$75^{* *}$ This report demonstrates that target deletion of interest gene in microglia by using $\mathrm{Cx} 3 \mathrm{cr} 1$-Cre or $\mathrm{Cx} 3 \mathrm{crl}$-CreER mice is very useful for the analyses of microglial function.

79* The authors demonstrate that replacement with wild type microglia in Mecp $2^{-/-}$ mice attenuates the pathology of a mouse model of Rett syndrome, an autism spectrum disorder induced by $M E C P 2$ mutation. Decreased phagocytic activity in Mecp $2^{-/-}$microglia seems to relate to the symptons. This provides an important clue that phagocytic function of microglia would be indispensable for normal brain development and function. 


\section{Figure legend}

Figure 1. Bidirectional functions of microglia in the developing brain. Microglia are generated in the yolk sac from E7.5 in mice (a) and migrate out of the sac, infiltrating the neuroepithelium through ventricles, meningeal tissue, or vasculature (b, red dotted arrows). Microglia eliminate unnecessary neural progenitor cells and may also support neurogenesis ( $\mathrm{b}$ and $\mathrm{c}$, red and blue arrows respectively). Progenitor cells start differentiating into neurons and migrate to their final position ( $\mathrm{c}$ and $\mathrm{d}$, green dotted arrows), where microglia support the survival of neurons and eliminate unnecessary ones (blue and red arrows, respectively). Neurons then project axons, and microglia transiently accumulate along the axonal tract in the postnatal period (d). How microglia accumulate along the axon largely unknown (red dotted arrow). Microglia may affect axonal growth/survival of neurons and engulf degenerating or pruned axon materials (blue and red arrows, respectively). Microglia distribute in the brain during neural circuit formation ( $\mathrm{d}$ to e, red dotted arrows) and survey their environment, frequently contacting synapses (e), and engulfing those that are unnecessary. This act may control homeostasis of neural activity. VZ, ventricular zone; SVZ, subventricular zone; ?, unknown or indicating that this has not been completely proved in vivo. 


\section{Table 1}

Molecules involved in developmental functions of microglia

\begin{tabular}{ll}
\hline Process & Paradigm \\
\hline
\end{tabular}

Initial differentiation / proliferation of microglia in vivo

Migration into the CNS

in vivo

in vivo

in vivo

in vivo

in vivo

Cell death induction

in vitro

in vitro

in vitro

in vivo

Cell survival

in vivo

Phagocytosis of neurons

in vivo

in vitro

Axonal growth

in vitro

Phagocytosis of axons

in vivo

Synapse elimination

in vivo

in vivo 